\title{
Armazenamento úmido e seco de rosas cortadas ${ }^{(1)}$
}

\author{
GLÁUCIA MORAES DIAS(2)*; JOSÉ MARIA MONTEIRO SIGRIST(3); \\ PATRICIA CIA ${ }^{(2)}$ e SYLVIO LUÍS HONÓRIO(4)
}

\begin{abstract}
RESUMO
Rosa sp. é o principal gênero de flor de corte comercializado no Brasil e no mundo, sendo que para manutenção da qualidade floral, as hastes devem ser armazenadas à $1{ }^{\circ} \mathrm{C}$. No entanto, ainda há dúvidas quanto à eficiência do armazenamento úmido associado à refrigeração antes da comercialização. Com o objetivo de avaliar a viabilidade do armazenamento de rosa 'Avalanche' em água ou a seco, hastes foram armazenadas por 28 dias a $1 \pm 2{ }^{\circ} \mathrm{C} / 95 \%$ UR. Semanalmente, estas hastes foram transferidas para $25^{\circ} \mathrm{C}$ / 70\% UR e mantidas em vasos contendo água de torneira. Observou-se que o decréscimo da qualidade da rosa foi inversamente proporcional ao tempo de armazenamento em ambos os tratamentos. Apesar do armazenamento com a base na água poder ocorrer por até 21 dias, quando é necessário armazenar rosas por uma ou duas semanas, o armazenamento refrigerado a seco é mais econômico e viável.
\end{abstract}

Palavras-chave: pós-colheita, temperatura baixa, qualidade da flor, Botrytis cinerea.

\begin{abstract}
Wet and dry storage of cut roses

Rosa sp. is the leading cut flower commercialized in Brazil and in the world; and to maintain stem quality it should be stored at $1{ }^{\circ} \mathrm{C}$, however, there are still doubts about the storage of roses, especially whether the stems should be cold stored in water before marketing. In order to assess the feasibility of wet and dry storage, rose stems, 'Avalanche' were stored for 28 days in both conditions at $1 \pm 2{ }^{\circ} \mathrm{C} / 95 \% \mathrm{RH}$. Weekly stems batches were transferred to $25^{\circ} \mathrm{C} / 70 \% \mathrm{RH}$ and kept in vessels containing tap water for postharvest assessment. It was observed that the decrease in the quality of the roses was inversely proportional to the storage time in both treatments. Although wet storage can occur for up to 21 days, when you need to store roses for a week or two, cold storage in dry condition is advised since it is more economical and viable for the producer.
\end{abstract}

Keywords: postharvest, low temperature, flower quality, Botrytis cinerea.

\section{INTRODUÇÃO}

A floricultura brasileira constitui-se em um dos segmentos mais dinâmicos do agronegócio brasileiro contemporâneo, exibindo indicadores de crescimento significativos, tanto em termos de número de produtores quanto em área cultivada. Este fato fica evidente ao se analisar o comércio de flores e plantas ornamentais no Brasil, o qual movimenta, anualmente, o valor global médio de R $\$ 5$ bilhões (JUNQUEIRA e PEETZ, 2014; NEVES e PINTO, 2015).

Para que a comercialização seja bem sucedida, é importante a manutenção da qualidade pós-colheita de flores de corte, de modo que todo o esforço empregado durante a produção seja compensado no momento da venda do produto. Neste cenário, destaca-se o manejo póscolheita, principalmente a utilização do armazenamento refrigerado, que auxilia a manutenção da qualidade das hastes florais, por reduzir os processos metabólicos, principalmente taxa respiratória, produção de etileno e transpiração. Esta técnica por ser empregada por via úmida ou seca, sendo esta última, geralmente, utilizada para transporte por longos períodos (DIAS-TAGLIACOZZO e MOSCA, 2007). No armazenamento a seco, as hastes não entram em contato com meio aquoso; no entanto, deve ocorrer em atmosfera com umidade relativa em torno de 90\% (HALEVY e MAYAK, 1981; NOVAK et al., 1991). Salienta-se, ainda, que durante a comercialização no consumidor final, as hastes deverão ser mantidas em água independente do armazenamento e transporte terem sido úmido ou seco.

As espécies do gênero Rosa $s p$. correspondem às principias flores de corte comercializadas no Brasil e no mundo, sendo que para manutenção de sua qualidade as hastes devem ser armazenadas a $1{ }^{\circ} \mathrm{C}$ (REID, 2004; SEBRAE, 2015). As flores são órgãos efêmeros, de modo que a redução da perecibilidade das mesmas é um desafio para a conservação pós-colheita. Durante a vida de vaso, muitos fatores contribuem para diminuição da qualidade da flor, entre eles estão intensidade luminosa, temperatura, ocorrência de patógenos e as relações hídricas. No caso das rosas em que a temperatura de armazenamento já é estabelecida para a $1{ }^{\circ} \mathrm{C}$, as preocupações maiores são para evitar o estresse hídrico, pois este ocasiona a falha na abertura floral, murcha das flores e folhas (MACNISHA et al., 2009; REID e JIANG, 2012), e a presença do fungo

\footnotetext{
(1) Recebido em 16/06/2016 e aprovado em 26/08/2016

(2) Instituto Agronômico (IAC), Jundiaí-SP, Brasil. *Autor correspondente: glaucia@iac.sp.gov.br

(3) Instituto de Alimentos (ITAL), Campinas-SP, Brasil.

(4) Universidade Estadual de Campinas (UNICAMP), Faculdade de Engenharia Agrícola. Campinas-SP, Brasil.
} 
Botrytis cinerea, causador de sérios danos na pós-colheita de rosas (FAVERO et al., 2015), o qual danifica as pétalas e apresenta esporulação visível (DROBY e LICHTER, 2007). Ainda há dúvidas quanto ao armazenamento de rosas, especialmente no que se refere à utilização da via seca ou úmida antes da comercialização. Assim, o objetivo deste trabalho foi avaliar a longevidade de hastes de rosas 'Avalanche', após o armazenamento refrigerado a seco e úmido.

\section{MATERIAL E MÉTODOS}

Hastes de rosa 'Avalanche' em estádio de botão, provenientes de Andradas/SP, foram colhidas, selecionadas pelo produtor, colocadas em água e, na sequência, transportadas para o Laboratório de Tecnologia PósColheita. Nenhuma manipulação foi realizada, pois se pretendeu usar a seleção e as condições de armazenamento realizadas pelo produtor. No laboratório, as hastes foram divididas em dois lotes, os quais foram mantidos a $1 \pm 2$ ${ }^{\circ} \mathrm{C} / 95 \%$ UR: metade das hastes teve a base imersa em água de torneira e a outra metade foi armazenada a seco na posição horizontal. As hastes foram mantidas nestas condições por até 28 dias. Semanalmente, hastes mantidas em armazenamento seco e úmido, tiveram suas bases cortadas em $5 \mathrm{~cm}$, transferidas para vasos com água potável e mantidas a $25{ }^{\circ} \mathrm{C} / 70 \%$ UR até senescerem. Com a finalidade de se elaborar um critério de notas para avaliações de manutenção da qualidade, foram selecionados os principais sinais de senescência, considerando brilho, turgescência das flores e abertura floral, sendo estas observações que permitiram o estabelecimento do critério de notas descrito na tabela 1 , o qual foi usado para as avaliações da qualidade da flor. Para a constatação de que o fungo visualizado era o Botrytis cinerae utilizou o método de isolamento indireto (ALFENAS et al., 2007). As avaliações foram realizadas em dias alternados, seguida de

Tabela 1. Avaliação da qualidade de rosas.

Table 1. Roses quality assessment.

\begin{tabular}{|c|c|c|}
\hline & Nota & Definição \\
\hline \multirow{5}{*}{$\frac{\grave{O}}{I}$} & 10 & Excelente (sem danos aparente) \\
\hline & 8 & Bom (pétala externa danificada, mas com brilho, botão fechado) \\
\hline & 6 & Regular (início da perda de brilho, flor aberta) \\
\hline & 4 & Ruim (pétalas sem brilho, início de sinais de perda de turgescência) \\
\hline & 2 & Péssimo - descarte (pétalas sem brilho, flor com pétalas visivelmente murchas) \\
\hline \multirow{10}{*}{ 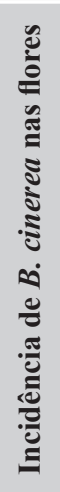 } & 1 & $0 \%$ \\
\hline & 2 & $0-2 \%$ \\
\hline & 3 & $3-5 \%$ \\
\hline & 4 & $6-10 \%$ \\
\hline & 5 & $11-15 \%$ \\
\hline & 6 & $16-25 \%$ \\
\hline & 7 & $26-50 \%$ \\
\hline & 8 & $51-75 \%$ \\
\hline & 9 & $76-99 \%$ \\
\hline & 10 & $100 \%$ \\
\hline
\end{tabular}

troca de água nos vasos.

O delineamento estatístico foi inteiramente casualizado com quatro repetições e 10 hastes como unidade experimental. Os resultados das avaliações de qualidade da flor foram submetidos à análise de variância com o programa SISVAR ${ }^{\circledR}$ (FERREIRA, 2011), utilizando o teste de Tukey ao nível de 5\% de probabilidade para a comparação entre os tratamentos.

\section{RESULTADOS E DISCUSSÃO}

Hastes submetidas ao armazenamento úmido não perderam qualidade durante 7 ou 14 dias à $1{ }^{\circ} \mathrm{C}$ em UR de $95 \%$. No entanto, as hastes armazenadas a seco, nos mesmos períodos e condições, apresentaram sinais visíveis de murcha ao final destes tratamentos (dia $0)$. Entretanto, após um dia com a base na água estas recuperaram a turgescência e não apresentaram diferenças significativas de qualidade quando comparadas com as armazenadas em água.

Essa qualidade semelhante da flor, independente do armazenamento ser úmido ou seco, também foi observada quando esse período pós armazenamento se estendeu por 5 e 7 dias em água à $25{ }^{\circ} \mathrm{C}$ em UR de $70 \%$ 


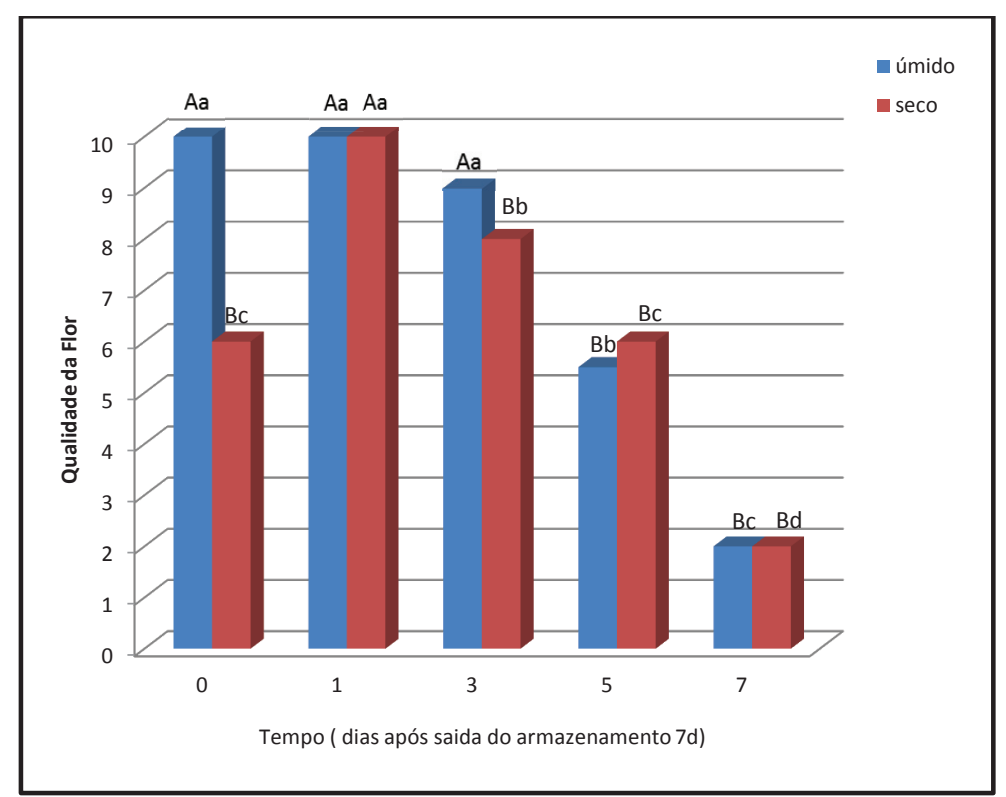

Figura 1. Qualidade da flor de rosas 'Avalanche' após armazenamento a $1{ }^{\circ} \mathrm{C} / 95 \% \mathrm{UR}$, com a base imersa em água (úmido) ou não (a seco), por sete dias seguido por mais sete dias a $25^{\circ} \mathrm{C} / 70 \% \mathrm{UR}$. (Letras maiúsculas comparam os tratamentos no mesmo dia e minúsculas cada tratamento ao longo do tempo).

Figure 1. Flower quality of 'Avalanche' roses after wet or dry storage at $1{ }^{\circ} \mathrm{C} / 95 \% \mathrm{RH}$ for seven days followed by seven days at $25{ }^{\circ} \mathrm{C} / 70 \% \mathrm{RH}$. (Capital letters compare the treatments on the same day and lower case letters compare each treatment over time).

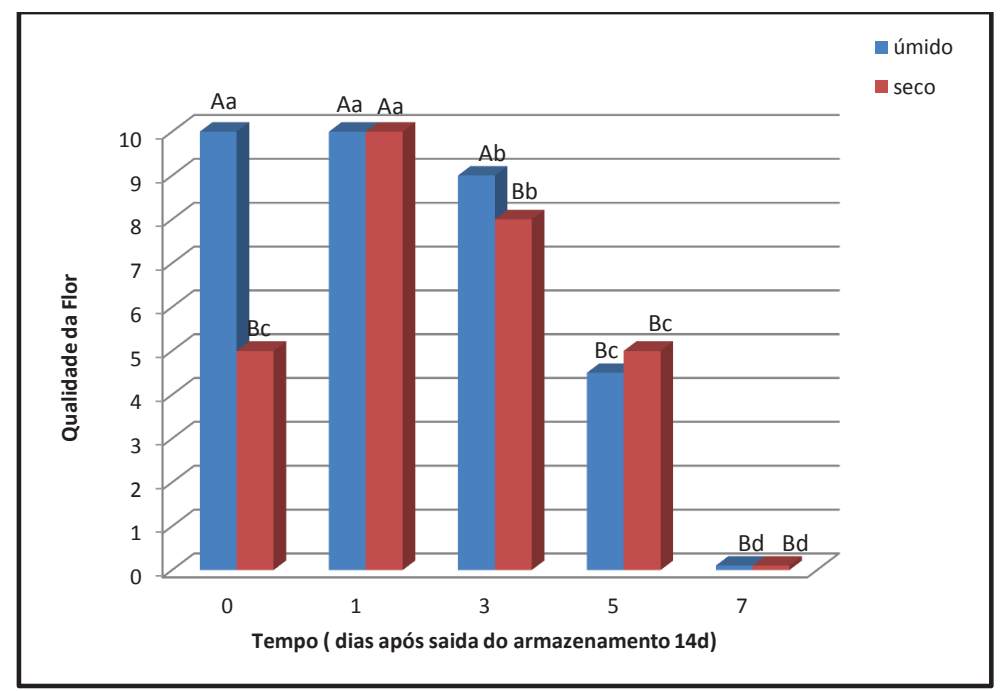

Figura 2. Qualidade da flor de rosas 'Avalanche' após armazenamento a $1{ }^{\circ} \mathrm{C} / 95 \% \mathrm{UR}$, com a base imersa em água ( úmido) ou não (a seco), por quatorze dias seguido por mais sete dias a $25^{\circ} \mathrm{C} / 70 \%$ UR. (Letras maiúsculas comparam os tratamentos no mesmo dia e minúsculas cada tratamento ao longo do tempo).

Figure 2. Flower quality of 'Avalanche' roses after wet or dry storage at $1{ }^{\circ} \mathrm{C} / 95 \%$ RH for fourteen days followed by seven days at $25^{\circ} \mathrm{C} / 70 \% \mathrm{RH}$. (Capital letters compare the treatments on the same day and lower case letters compare each treatment over time). 
(Figuras 1 e 2).

A partir de 21 dias de armazenamento, observou-se diferenças significativas entre as hastes armazenadas a seco e úmido, de modo que hastes armazenadas a seco ao terem suas bases colocadas em água, não conseguiam recuperar a turgescência, perdendo rapidamente a durabilidade comercial. No entanto, as hastes com a base na água mantidas à $25^{\circ} \mathrm{C}$ em UR de $70 \%$ até 5 dias após o armazenamento apresentaram valores semelhantes às armazenadas nas mesmas condições por 14 dias (Figuras 2 e 3 ).

Ao final do armazenamento à $1{ }^{\circ} \mathrm{C}$ em UR de $95 \%$, por 28 dias, as hastes armazenadas à seco não apresentaram qualidade comercial e as armazenadas com a base na água a partir do terceiro não apresentavam mais qualidade

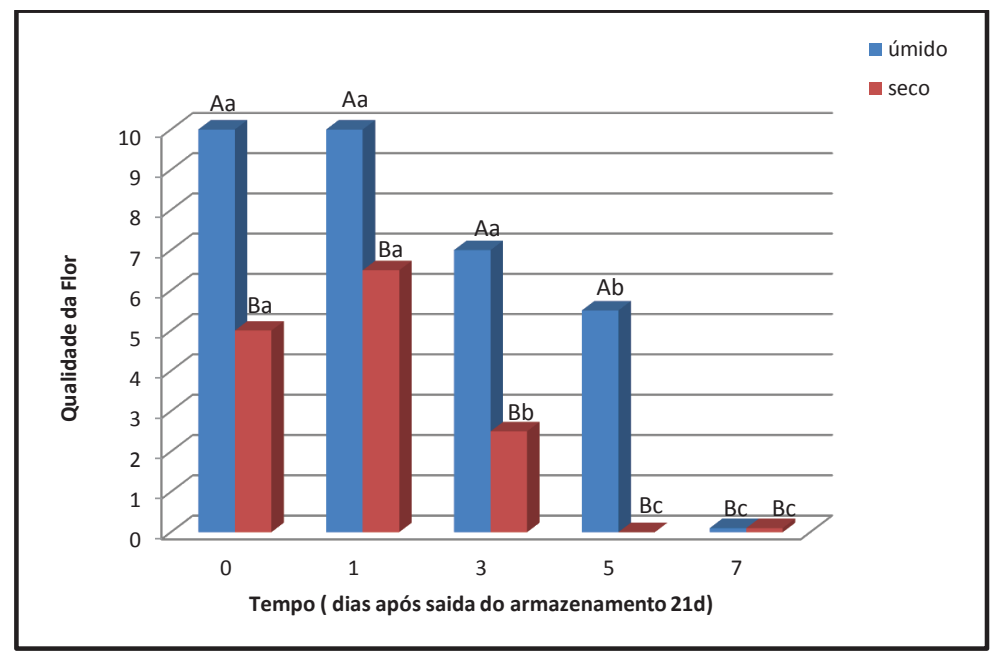

Figura 3. Qualidade da flor de rosas 'Avalanche' após armazenamento a $1{ }^{\circ} \mathrm{C} / 95 \% \mathrm{UR}$, com a base imersa em água ( úmido) ou não (a seco), por vinte e um dias seguido por mais sete dias a $25^{\circ} \mathrm{C} / 70 \%$ UR. (Letras maiúsculas comparam os tratamentos no mesmo dia e minúsculas cada tratamento ao longo do tempo).

Figure 3. Flower quality of 'Avalanche' roses after wet or dry storage at $1{ }^{\circ} \mathrm{C} / 95 \%$ RH for twenty-one days followed by seven days at $25^{\circ} \mathrm{C} / 70 \% \mathrm{RH}$. (Capital letters compare the treatments on the same day and lower case letters compare each treatment over time).

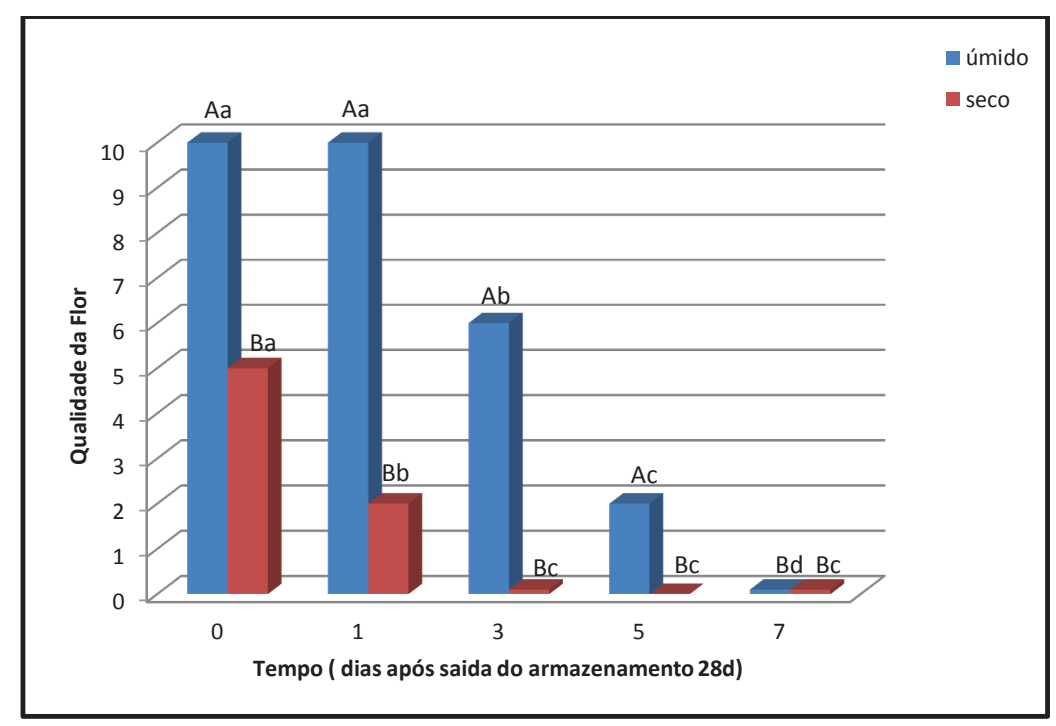

Figura 4. Qualidade da flor de rosas ‘Avalanche’ após armazenamento a $1{ }^{\circ} \mathrm{C} / 95 \% \mathrm{UR}$, com a base imersa em água ( úmido) ou não (a seco), por vinte e oito dias seguido por mais sete dias a $25^{\circ} \mathrm{C} / 70 \% \mathrm{UR}$. (Letras

maiúsculas comparam os tratamentos no mesmo dia e minúsculas cada tratamento ao longo do tempo).

Figure 4. Flower quality of 'Avalanche' roses after wet or dry storage at $1{ }^{\circ} \mathrm{C} / 95 \%$ RH for twenty-eight days followed by seven days at $25^{\circ} \mathrm{C} / 70 \% \mathrm{RH}$. (Capital letters compare the treatments on the same day and lower case letters compare each treatment over time). 
comercial (Figura 4).

O material recebido do produtor chegou com baixa incidência de Botrytis cinerea e, portanto, esse fungo não influenciou na qualidade das rosas nesses tratamentos.

A turgescência nas plantas intactas e flores colhidas é dependente de um balanço entre a utilização e o fornecimento de água. Esse balanço hídrico envolve processos fisiológicos, sendo a refrigeração uma forma de retardar esses processos e, consequentemente, prolongar a longevidade das flores colhidas (DIAS-TAGLIACOZZO et al., 2006). Observou-se que as hastes armazenadas sobrefrigeração a seco, apesar de perderem água durante o armazenamento, estas provavelmente não sofreram danos fisiológicos quando o período de armazenamento foi inferior a 14 dias, pois a turgescência foi recuperada quando suas bases retornaram para água. No entanto, após 21 dias de armazenamento, devem ter ocorrido danos nos processos fisiológicos, pois o aspecto de murcha se manteve. Já as flores armazenadas em água recuperaram a turgescência no dia 1, independente do período de armazenamento; no entanto, o armazenamento por 28 dias não é recomendado, pois a qualidade foi perdida rapidamente.

Apesar do armazenamento com a base na água poder ocorrer por períodos mais longos, quando o produtor necessita armazenar suas flores por uma ou duas semanas, o armazenamento e transporte refrigerado a seco é mais econômico e viável, visto que o volume de flores armazenados por $\mathrm{m}^{3}$ é maior nessas condições, além de não ser necessária a alocação em recipientes com água, obtendo-se resposta comercial semelhante às flores mantidas em água.

\section{CONCLUSÕES}

- O decréscimo da qualidade das rosas foi inversamente proporcional ao tempo de armazenamento;

- O armazenamento refrigerado de rosas por 7 e 14 dias é eficiente para armazenamento tanto à seco ou em água.

- O armazenamento refrigerado de rosas por 21 dias é eficiente somente para armazenamento em água.

- O período de 28 dias não é recomendado para o armazenamento refrigerado de rosas.

\section{AGRADECIMENTOS}

À FAPESP pelo incentivo durante o desenvolvimento do projeto de pesquisa.

\section{REFERÊNCIAS}

ALFENAS, A.C.; FERREIRA, F.A.; MAFIA, R.G.; GONÇALVES, R.C. Isolamento de fungos fitopatogênicos. In: ALFENAS, A.C.; MAFIA, R.G. Métodos em
Fitopatologia. 1ed. Viçosa: Editora UFV, 2007. p.53-91,

DIAS-TAGLIACOZZO, G.M.; MOSCA, J.L. Pós-colheita de flores e Folhagem. Revista Brasileira de Horticultura Ornamental, v.13, p.209-219, 2007.

DIAS-TAGLIACOZZO, G.M; FINGER, F.L.; BARBOSA, J.G. Fisiologia pós-colheita de flores de corte. Revista Brasileira de Horticultura Ornamental, v.11, p.89-99, 2006.

DROBY, S.; LICHTER, A. Post-harvest Botrytis infection: etiology, development and management. In: ELAD, Y.; WILLIAMSON, B.; TUDZYNSKI, P.; DELEN, N. Botrytis: biology, pathology and control. 1 ed. Dordrencht: Springer, 2007. p.349-68.

FAVERO, B.T.; BENATO, E.A.; DIAS, G.M.; CIA, P. Gibberellic acid, ozone and 1-methylcyclopropene on the gray mold control in 'Avant Garde' rose. Acta Horticulturae, v.1060, p.177-182, 2015.

FERREIRA, D.F. Sisvar: a computer statistical analysis system. Ciência e Agrotecnologia, v.35, p.1039-1042, 2011.

HALEVY, A.H.; MAYAK, S. Senescence and postharvest physiology of cut flowers- part 2. In: JANICK, J. (ed.). Horticultural Reviews. Westport: AVI Publishing, v.3, 1981. p.59-143.

JUNQUEIRA, A.H.; PEETZ, M.S. O setor produtivo de flores e plantas ornamentais do Brasil, no período de 2008 a 2013: atualizações, balanços e perspectivas. Revista Brasileira de Horticultura Ornamental, v.2, p.115-120, 2014.

MACNISHA, A.J.; THEIJE, A.; REID, M.S. An alternative postharvest handling strategy for cut flowers - Dry handling after harvest. Acta Horticulturae, n.847, p.215-221, 2009

NEVES, M.F.; PINTO, M.J.A. Mapeamento e quantificação da cadeia de flores e plantas ornamentais do Brasil. São Paulo: OCESP, 2015. 122p.

NOVAK, J.; GOSZCZYNSKA, M.D.; RUDINICKI, R.M. Storage of cut flower and ornamental plants: present status and future prospects. Postharvest News and information. 
Research Institute of Pomology and Floriculture, v.2, p.255-260, 1991.

REID, M.S.; JIANG, C.Z. Postharvest biology and technology of cut flowers and potted plants. Horticultural Reviews, v.40, p.1-54, 2012.
REID, M.S. Roses, Spray Rose, Sweetheart Rose: Recommendations for Maintaining Postharvest Quality. 2004. Disponível em: <http://postharvest.ucdavis.edu/ pfornamentals/Roses/>. Acesso em: 22 de fevereiro de 2016.

SEBRAE. Balanço Comercial da Floricultura Brasileira. Flores e plantas ornamentais do Brasil (série estudos mercadológicos), São Paulo, v.2, p.73-77, 2015. 